\title{
Efficient Force Calculations Based on Continuum Sensitivity Analysis
}

\author{
Dong-Hun Kim ${ }^{1}$, David Lowther ${ }^{2}$ and Jan K. Sykulski ${ }^{1}$ \\ ${ }^{1}$ School of Electronics and Computer Science, University of Southampton \\ Southampton, SO17 1BJ, UK, E-mail: dh29kim@dreamwiz.com, jks@soton.ac.uk \\ ${ }^{2}$ Department of Electrical Engineering, McGill University \\ Montreal, Quebec, H3A 2A7Canada, E-mail: david.lowther@mcgill.ca
}

\begin{abstract}
Using Continuum Design Sensitivity Analysis (CDSA), which is based on a Virtual Work Principle, equations have been derived for calculating forces without the need to solve the adjoint system. The resultant expressions are similar to the Maxwell Stress Tensor but have an important advantage of the integration taking place on the surface of material rather than in the air outside. Implementation of the scheme leads to very efficient calculations and improved accuracy.
\end{abstract}

\section{INTRODUCTION}

Computation of forces in electromagnetic systems is recognised to be a difficult problem. Most commonly used approaches develop a Virtual Work Principle (VWP) or Maxwell Stress Tensor (MST). Since force may be considered as the sensitivity of stored energy to changes in geometry, the computation of force through a sensitivity based process should be possible. The work of Coulomb [1] could be viewed as an implementation of a discrete sensitivity approach. However, force calculation should be independent of the numerical approach used to compute the fields. Thus this paper focuses on using the continuum sensitivity analysis [2] as a new means of calculating forces efficiently.

\section{FORMULATION}

Due to space restrictions only the linear formulation will be presented briefly. We start with the sensitivity formula:

$$
\frac{d O}{d \mathbf{p}}=\int_{Y}\left[\left(v_{1}-v_{2}\right) \nabla \times \mathbf{A}_{1} \cdot \nabla \times \lambda_{2}+\left(\mathbf{M}_{p 1}-\mathbf{M}_{p 2}\right) \cdot \nabla \times \lambda_{2}-\left(\mathbf{J}_{1}-\mathbf{J}_{2}\right) \cdot \lambda_{2}\right] V_{n} d \Gamma
$$

The objective function should be defined in terms of coenergy of the system, and for a linear system we can write

$$
O=2 W_{m}^{\prime}=\int_{\Omega} \mathbf{B} \cdot d \mathbf{H} d \Omega=\int_{\Omega} \mathbf{J} \cdot \mathbf{A} d \Omega
$$

Thus

$$
\mathbf{f}_{1}=2 \partial W_{m}^{\prime} / \partial \mathbf{A}=\int_{\Omega} \partial(\mathbf{J} \cdot \mathbf{A}) / \partial \mathbf{A} d \Omega=\int_{\Omega} \mathbf{J} d \Omega
$$

and $\mathbf{A}=\boldsymbol{\lambda}$, which means that the adjoint system is not needed!

The expression for force may now be written as

$$
\mathbf{F}=\frac{1}{2} \frac{d\left(2 W_{m}^{\prime}\right)}{d \mathbf{p}}=\frac{1}{2} \int_{y}\left[\left(v_{1}-v_{2}\right) \nabla \times \mathbf{A}_{1} \cdot \nabla \times \boldsymbol{\lambda}_{2}+\left(\mathbf{M}_{p 1}-\mathbf{M}_{p 2}\right) \cdot \nabla \times \boldsymbol{\lambda}_{2}-\left(\mathbf{J}_{1}-\mathbf{J}_{2}\right) \cdot \boldsymbol{\lambda}_{2}\right] \mathbf{n} d \Gamma
$$

The first term looks like the Maxwell Stress Tensor, but is applied to the surface of the material, rather than air outside:

$$
\begin{aligned}
\mathbf{F} & =\frac{1}{2} \int_{\gamma}\left[\left(v_{1}-v_{2}\right) \nabla \times \mathbf{A}_{1} \cdot \nabla \times \boldsymbol{\lambda}_{2}\right] \mathbf{n} d \Gamma \\
& =\frac{1}{2} \int_{\gamma}\left(v_{1}-v_{2}\right)\left[B_{2 n}^{2}+\frac{v_{2}}{v_{1}} B_{2 t}^{2}\right] \mathbf{n} d \Gamma
\end{aligned}
$$

The second term in (4) describes the force on a permanent magnet, whereas the last term is the $\mathbf{J} \times \mathbf{B}$ component. Hence in a linear case the implementation is particularly efficient.
EXAMPLE

Consider a simple $\mathrm{C}$-core magnet. Forces on a plunger have been calculated based on VWP, MST and using the internal MST commands of the commercial codes OPERA and MagNet (Fig. 1). A normalized error (with relation to VWP) is shown in Fig. 2.

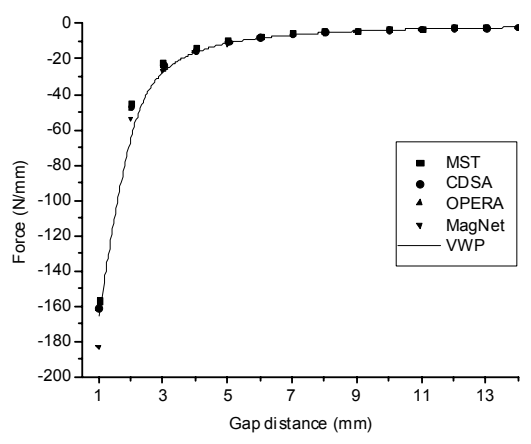

Fig. 1. Force versus distance in a C-core magnet.

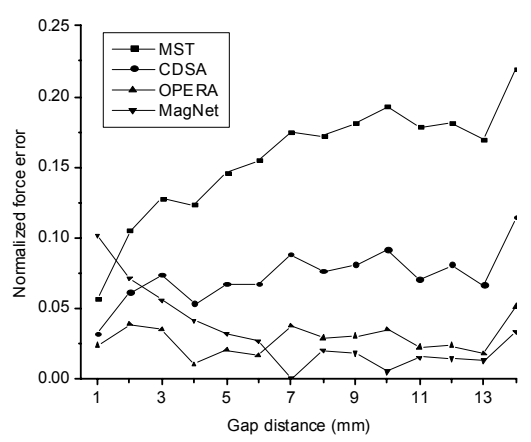

Fig. 2. Normalised errors.

The CDSA formulation improves accuracy of the standard Maxwell Stress Method and is much easier to implement as integration is carried out over the surface and not in the air outside. It is also more efficient than Virtual Work Principle as only one solution is required. In the final paper the approach will be extended to include non-linear effects.

\section{REFERENCES}

[1] J.L. Coulomb, and G. Meunier, "Finite element implementation of virtual work principle to magnetic or electric force and torque computation," IEEE Trans Magn, vol. 20, pp. 1894-6, Sept 1984.

[2] Dong-Hun Kim, Se-Hee Lee, et al., "Derivation of a general sensitivity formula for shape optimization of $2 \mathrm{D}$ magnetostatic systems by continuum approach," IEEE Trans Magn, vol. 38, pp. 1125-8, Mar 2002. 